Original article

\title{
A comparison of the malnutrition screening tools, MUST, MNA and bioelectrical impedance assessment in frail older hospital patients
}

\author{
Adrian Slee ${ }^{\mathrm{a}, *}$, Deborah Birch ${ }^{\mathrm{b}}$, David Stokoe ${ }^{\mathrm{c}}$ \\ ${ }^{a}$ School of Life Sciences, Brayford Pool Campus, Lincoln, Lincolnshire LN6 7TS, UK \\ ${ }^{\mathrm{b}}$ Lincoln Hospital, ULHT, Lincoln, Lincolnshire LN2 5QY, UK \\ ' St Barnabas Hospice, ULHT, Lincoln, Lincolnshire LN2 1RE, UK
}

\section{A R T I C L E I N F O}

Article history:

Received 2 October 2013

Accepted 22 April 2014

\section{Keywords:}

Malnutrition

Frailty

MUST

MNA

Bioelectrical impedance assessment

\begin{abstract}
S U M M A R Y
Background E aims: This cohort study aimed to investigate and compare the ability to predict malnutrition in a group of frail older hospital patients in the United Kingdom using the nutritional risk screening tools, MUST (malnutrition universal screening tool), MNA-SF ${ }^{\circledR}$ (mini nutritional assessment-short form) and bioelectrical impedance assessment (BIA) of body composition.

Methods: MUST and MNA-SF was performed on 78 patients (49 males and 29 females, age: $82 \mathrm{y} \pm 7.9$, body mass index (BMI): $25.5 \mathrm{~kg} / \mathrm{m}^{2} \pm 5.4$ ), categorised by nutritional risk, and statistical comparison and test reliability performed. BIA was performed in 66 patients and fat free mass (FFM), fat mass (FM) and body cell mass $(\mathrm{BCM})$ and index values $\left(\mathrm{kg} / \mathrm{m}^{2}\right)$ calculated and compared against reference values.

Results: MUST scored 77\% patients 'low risk', 9\% 'medium risk' and 14\% 'high risk', compared to MNA-SF categorisation: $9 \%, 46 \%$ and $45 \%$, respectively $(P<0.000001)$. Reliability assessment found poor reliability between the screening tools (coefficient, $r=0.4$ ). Significant positive correlations were found between most variables $(P<0.05-<0.001)$; although females exhibited greater variation. FFM index analysis found $40 \%$ of males low/depleted, $21 \%$ borderline/at risk with $96 \%$ categorised by MNA-SF as either malnourished or at risk (MUST-35\%). 29\% males had low FM index and all appropriately classified by MNA-SF. 30\% females had low FFM index or borderline, MNA-SF screening appropriately categorised $86 \%$ (compared to MUST-29\%).

Conclusions: This preliminary data may have significant clinical implications and highlights the potential ability of the MNA-SF and BIA to accurately assess malnutrition risk over MUST in frail older hospital patients.

(C) 2014 Elsevier Ltd and European Society for Clinical Nutrition and Metabolism. All rights reserved.
\end{abstract}

\section{Introduction/background}

Malnutrition is a serious condition associated with increased morbidity and mortality and is particularly relevant in older people [1-3]. Older people may be at increased risk due to physiological alterations in body composition during ageing (e.g. the loss of skeletal muscle mass, 'sarcopenia', and associated muscle protein), and reduction in appetite (e.g. 'the anorexia of ageing') [4-7]. In addition, complexity is added with the occurrence of acute and chronic disease and pathological frailty syndrome producing concurrent and overlapping symptoms of cachexia, sarcopenia and undernutrition [8-11].

\footnotetext{
* Corresponding author.

E-mail addresses: aslee@lincoln.ac.uk (A. Slee), deborah.birch@ulh.nhs.uk (D. Birch), david.stokoe@stbarnabashospice.co.uk (D. Stokoe).
}

Specific guidelines to screen for malnutrition/nutritional risk have been developed by ESPEN [12] and, currently, in the United Kingdom (UK) the malnutrition universal screening tool (MUST), endorsed by BAPEN, is utilised in all hospitals and care homes [13]. MUST formulates a risk of malnutrition score based upon current body mass index (BMI), known weight loss and the presence of acute disease/no nutritional intake for 5 days [12,13]. This score partially forms the basis upon which clinical and dietetic decisions are formulated. The mini-nutritional assessment (MNA), designed specifically and validated for older people, has a full version of 18 questions and a short-form screening version $\left(\mathrm{MNA}_{\mathrm{S}} \mathrm{F}^{\circledR}\right.$ ) of 6 questions [12,14-16]. The MNA-SF has similar questions to the MUST with additional questions on neuropsychological functional status, physical mobility and food intake. Scoring works in the opposite direction to the MUST with a lower score indicating a higher risk of malnutrition. The BMI classifications for the MUST are principally based upon the World Health Organisation (WHO) BMI classifications with normal at $>20 \mathrm{~kg} / \mathrm{m}^{2}$ (score-0), 'at risk' $18.5-20$ 
(1) and 'high risk'/'malnourished' $<18.5$ (2), whereas for the MNA it is has a graded classification with $<19$ (score-0), 19-21 (1), 21-23 (2) and $>23$ (3).

Bioelectrical impedance assessment (BIA) is a practical means of assessing body composition and nutritional status using body impedance data $[17,18]$. Specific prediction equations for estimating body compartments of interest (e.g. fat mass (FM) and fat free mass (FFM)) have been developed and validated using gold standard techniques such as dual-energy X-ray absorptiometry (DEXA) for specific healthy population groups, and BIA analyser manufacturers have also developed their own equations [17]. Other relevant body compartments of interest in relation to nutritional status include the body cell mass (BCM) component which is FFM minus extracellular water fluid (ECW). Further, specific indices (normalised for height, i.e. $\mathrm{kg} / \mathrm{m}^{2}$ ) of FM, FFM and BCM can be compared to reference ranges similar to the BMI. One issue of concern however, is that the BIA predictive equations may cause significant errors in older people and in pathological clinical states [17,18]; and that use of raw impedance data (i.e. the BIA vector method by Piccoli and Pastori) may have greater accuracy [18].

No studies were discovered comparing the utility of MUST, MNA-SF and BIA in frail older hospital patients. Therefore, the aims of this preliminary study were to assess the relative ability of the MUST and MNA-SF to predict malnutrition in frail older hospital inpatients; and to compare use of BIA as an additional method of assessing nutritional status.

\section{Methods}

\subsection{Participants and study design}

This cohort study was undertaken between September 2012 and May 2013 and recruits were from a purposive sampling from admissions to two hospital wards in Lincoln, UK specialising in care of frail older patients. Full ethical approval was obtained prior to study commencement, ethical guidelines followed and informed consent sought from all patients. Exclusion criteria from the study were: patients unable or unwilling to give informed consent, nil by mouth or tube fed. BIA measures were contraindicated in patients with defibrillation or cardiac pacemaker devices. The aim was to recruit 100-150 patients in-line with other similar studies; however the exclusion criterion of ability to consent and designated study time restraints dictated the current number.

\section{Nutritional assessment}

\subsection{MUST tool and MNA-SF ${ }^{\circledR}$ screening}

MUST and MNA-SF ${ }^{\circledR}$ screening was undertaken by clinical staff according to instructions and scores recorded. Scores were converted into categories for nutritional status using MUST and MNA$\mathrm{SF}^{\circledR}$ scoring criteria either 'low risk'/'normal'(0 points-MUST, 12-14 MNA-SF), 'medium risk/at risk' (1 point-MUST, 8-11 MNA-SF) and 'high risk'/'malnourished' ( $\geq 2$ points-MUST, 0-7 MNA-SF).

\subsection{Anthropometric measurements}

Height (m) and weight ( $\mathrm{kg}$ ) measurements were completed by clinical staff. In some cases height had to be estimated, e.g. height from demi-span. BMI was then calculated in $\mathrm{kg} / \mathrm{m}^{2}$.

\subsection{Bioelectrical impedance measurements}

BIA measurements were taken using a single-frequency (50 kHz) Maltron ${ }^{\circledR}$ 916S, bioelectrical impedance analyser
(Maltron International Ltd., Rayleigh, Essex, UK) using a standard hand-to-foot tetra-polar technique with participants in the supine position. Raw impedance measurements of resistance, $R$ and capacitance, $\mathrm{xC}$ in ohms were recorded at $50 \mathrm{kHz}$ frequency. FFM (kg) was calculated using the equation by Kyle et al. [19]. (see Appendices) Other estimations of FFM were also calculated and compared, including the BIA manufacturer (Maltron); but the Kyle equation was utilised for full data presentation based upon scatterplots of participant data against BMI and the general scientific consensus of the robust accuracy of equation. FM (kg) was calculated by subtraction of FFM from total body weight $(\mathrm{kg})$. The BCM (kg) was calculated using the Maltron (manufacturers) equation. FFM, FM and BCM indices $\left(\mathrm{kg} / \mathrm{m}^{2}\right)$ were calculated for all participants (FFMI, FMI and BCMI) and compared against reference data values from sources [20-22].

\subsection{Data analysis}

Data is presented as mean average measurements \pm SD with a range (minimum-maximum) and [median] values. Data is grouped into whole participant group, males and females, and where relevant into nutritional screening categories. Statistical analysis was performed using IBM SPSS Statistics, version 19, New York, USA. Ttests and Pearson correlations were used for normally distributed data and Mann-Whitney-U and Spearman correlations test for nonparametric data. McNemar pair comparison of malnutrition risk categorisation was performed on the 78 and 66 patient groups using a $2 \times 2$ contingency table, after categorising patients by low risk/normal, and combining medium risk/at risk and high risk/ malnourished groups (see Appendices, Suppl. Table 2A and B). Marginal frequencies, proportions were determined and significance value attained. Parallel-forms reliability analysis was performed to compare the MUST and MNA-SF scores and a reliability coefficient value $(r)$ attained. Categorical differences between male and female groups were analysed using Chi-squared and Fishers Exact testing. A $P$ value of $<0.05$ was considered statistically significant.

\section{Results}

\subsection{Participants}

The study group comprised of 78 participants in total (49 males and 29 females), predominantly Caucasian (all except two males), with a mean age of 82 years \pm SD 7.9 (age range: 62-96) (Table $1 \mathrm{~A}$ ). Due to the presence of a cardiac pacemaker nine patients were ineligible for BIA testing (69 remaining in total, 44 males and 25 females). Three participants (two male and one female) were further excluded from presentation of results as they had known hydration abnormalities and led to significant perturbations in FFM calculations. Table 1B shows all patient group FM, FFM and BCM (and indices) values.

\subsection{MUST and MNA-SF screening}

MUST and MNA-SF screening was completed for 78 patients in total. Fig. 1 shows the frequency of nutritional risk categorisation for patients using MUST and MNA-SF, as follows; MUST: 77\% 'low risk' (60/78), 9\% 'medium risk' (7/78) and 14\% 'high risk' (11/78); MNA-SF: 9\% 'normal' (7/78), 45\% 'at risk' (35/78) and 46\% 'malnourished' (36/78). Supplementary Table 1 in Appendices shows similar score patterns for the 66 BIA tested patients. McNemar statistical analysis of the MUST and MNA pair-wise grouping for patients (see Supplementary Table 2A and B) found highly significant differences in scoring patterns and discordance 
Table $1 \mathrm{~A}$

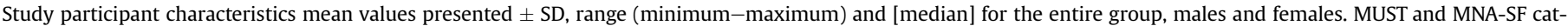
egorisation is also included with number and percentage of patients.

\begin{tabular}{|c|c|c|c|}
\hline & Group & Males & Females \\
\hline Number of participants, $n$ & 78 & 49 & 29 \\
\hline Age, years & $82.0 \pm 7.9(62-96)[83]$ & $80.7 \pm 7.8(62-92)[82]$ & $84.1 \pm 7.6(68-96)[85]$ \\
\hline Height, m & $1.68 \pm 0.10(1.37-1.85)[1.68]$ & $1.72 \pm 0.07(1.57-1.85)[1.74]$ & $1.62 \pm 0.10(1.37-1.78)[1.62]^{* * *}$ \\
\hline Weight, kg & $72.2 \pm 15.9(38.9-101.6)[71.0]$ & $73.7 \pm 15.8(42.8-101.0)[72.3]$ & $70.0 \pm 16.4(38.9-101.6)[69.9]$ \\
\hline $\begin{array}{l}\text { Body mass } \\
\quad \text { index, } \mathrm{kg} / \mathrm{m}^{2}\end{array}$ & $25.5 \pm 5.4(16.6-45.1)[24.9]$ & $24.6 \pm 4.9(16.6-35.2)[24.3]$ & $27.0 \pm 6.1(18.4-45.1)[26.7]^{*}$ \\
\hline MUST - 'Low risk' & $60(77 \%)$ & $36(74 \%)$ & $24(83 \%)$ \\
\hline MUST - 'medium risk' & $7(9 \%)$ & $4(8 \%)$ & $3(10 \%)$ \\
\hline MUST - 'High risk’ & $11(14 \%)$ & $9(18 \%)$ & $2(7 \%)$ \\
\hline MNA-SF Score & $8.0 \pm 2.8(2-14)[8]$ & $7.8 \pm 2.7(2-14)[8]$ & $8.0 \pm 2.9(2-14)[8]$ \\
\hline MNA-SF - 'normal' & $7(9 \%)$ & $3(6 \%)$ & $4(14 \%)$ \\
\hline MNA-SF - 'At risk' & $35(45 \%)$ & $24(49 \%)$ & $11(38 \%)$ \\
\hline MNA-SF - 'Malnourished' & $36(46 \%)$ & $22(45 \%)$ & $14(48 \%)$ \\
\hline
\end{tabular}

*Significantly different compared to female group $(P<0.05)$; ${ }^{* *}(P<0.01)$; ${ }^{* * *}(P<0.001)$

Table 1B

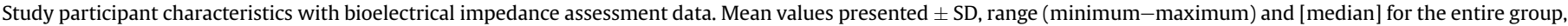
males and females.

\begin{tabular}{|c|c|c|c|}
\hline & Group & Males & Females \\
\hline Number of participants, $n$ & 66 & 42 & 24 \\
\hline Age, years & $82.1 \pm 7.5(62-96)[83]$ & $81.5 \pm 7.3(62-92)[82]$ & $83.2 \pm 7.7(68-96)[85]$ \\
\hline Height, m & $1.68 \pm 0.10(1.37-1.85)[1.68]$ & $1.72 \pm 0.07(1.57-1.85)[1.73]$ & $1.62 \pm 0.10(1.37-1.78)[1.62]^{* * *}$ \\
\hline Weight, kg & $73.4 \pm 14.8(42.8-101.6)[71.5]$ & $73.5 \pm 15.0(42.8-101)[73.5]$ & $73.3 \pm 14.9(49.8-101.6)[70.7]$ \\
\hline Body mass index, $\mathrm{kg} / \mathrm{m}^{2}$ & $26.0 \pm 5.4(16.6-45.1)[25.6]$ & $24.7 \pm 4.7(16.6-35.2)[24.4]$ & $\begin{array}{l}28.2 \pm 5.9(18.4-45.1) \\
{[28.3]^{* *}}\end{array}$ \\
\hline MNA-SF score & $8.1 \pm 2.8(2-14)[8]$ & $7.8 \pm 2.8(2-14)[8]$ & $8.7 \pm 2.7(5-14)[8.5]$ \\
\hline Fat free mass, $\mathrm{kg}$ & $50.0 \pm 9.3(31.7-72.7)[50.5]$ & $\begin{array}{l}53.4 \pm 8.8(37.5-72.7) \\
{[52.3]}\end{array}$ & $\begin{array}{l}44.2 \pm 7.2(31.7-58.7) \\
{[41.7]^{* * *}}\end{array}$ \\
\hline Fat free mass index, $\mathrm{kg} / \mathrm{m}^{2}$ & $17.62 \pm 2.46(13.22-23.49)[17.73]$ & $17.95 \pm 2.26(13.26-23.10)[17.86]$ & $17.07 \pm 2.67(13.22-23.49)[16.92]$ \\
\hline Fat mass, kg & $23.5 \pm 11.0(3.1-50.6)[21.1]$ & $\begin{array}{l}20.1 \pm 9.9(3.1-42.5) \\
{[18.9]}\end{array}$ & $29.3 \pm 10.4(12.2-50.6)[28.6]^{* * *}$ \\
\hline Fat mass index, $\mathrm{kg} / \mathrm{m}^{2}$ & $8.5 \pm 4.3(1.1-22.5)[7.6]$ & $6.8 \pm 3.4(1.1-14.0)[6.4]$ & $\begin{array}{l}11.3 \pm 4.1(3.8-22.5) \\
{[10.6]^{* * *}}\end{array}$ \\
\hline Body cell mass, kg & $26.6 \pm 5.2(17.0-37.5)[26.3]$ & $\begin{array}{l}28.1 \pm 5.6(17.0-37.5) \\
{[29.0]}\end{array}$ & $\begin{array}{l}24.0 \pm 3.3(18.1-30.1) \\
{[23.1]^{* * *}}\end{array}$ \\
\hline Body cell mass index, $\mathrm{kg} / \mathrm{m}^{2}$ & $9.4 \pm 1.5(6.3-13.0)[9.4]$ & $9.5 \pm 1.7(6.3-13.0)[9.5]$ & $9.2 \pm 1.2(7.4-11.7)[9.3]$ \\
\hline
\end{tabular}

*Significantly different compared to female group $(P<0.05)$; ${ }^{* *}(P<0.01)$; ${ }^{* * *}(P<0.001)$.

for the entire 78 patient group $(P=<0.000001)$ and the 66 BIA $(P=<0.000001)$. Furthermore, parallel forms reliability analysis indicated a high degree of variance between the two scales and the reliability correlation coefficient was 0.4 (i.e. $>0.90$ excellent, 0.80 0.89 -good and $0.70-0.79$-adequate etc.). Note: there were no significant differences in MUST and MNA scoring categorisation between males and females for the 78 and 66 patient groups.

\subsection{Body weight and body mass index}

Mean and ranges of body weight and BMI of patients can be seen in Table 1. The spread of male and female BMI and MNA-SF scores can be seen in Fig. 2. Correlations of body weight and MNA-SF score were; group $(r=0.42, P<0.001)$, males $(r=0.46, P<0.001)$ and females $(r=0.36, P=0.057)$, with lower weight correlating with a lower MNA-SF score. Correlations of BMI and MNA-SF score were; group $(r=0.52, P<0.001)$, males $(r=0.51, P<0.001)$, and females $(r=0.40, P<0.05)$.

\subsection{Fat free mass and fat free mass index}

FFM and FFMI for all 66 participants can be seen in Table 1B. Correlations of FFMI and BMI were; group $(r=0.614, P<0.0001)$, males $(r=0.787, P<0.0001)$, and females $(r=0.568, P<0.04)$, see

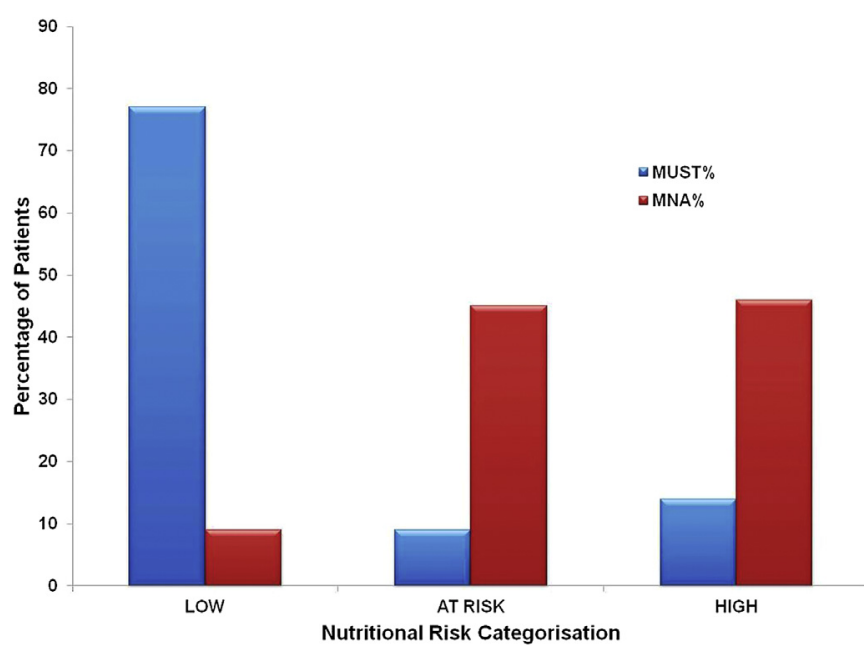

Fig. 1. Relative frequencies of nutritional risk categorisation of patients in percentages (\%). N.B. Low risk categorisation also equates to 'normal' by MNA-SF and high risk as 'malnourished'; and at risk as 'medium risk' by MUST. 


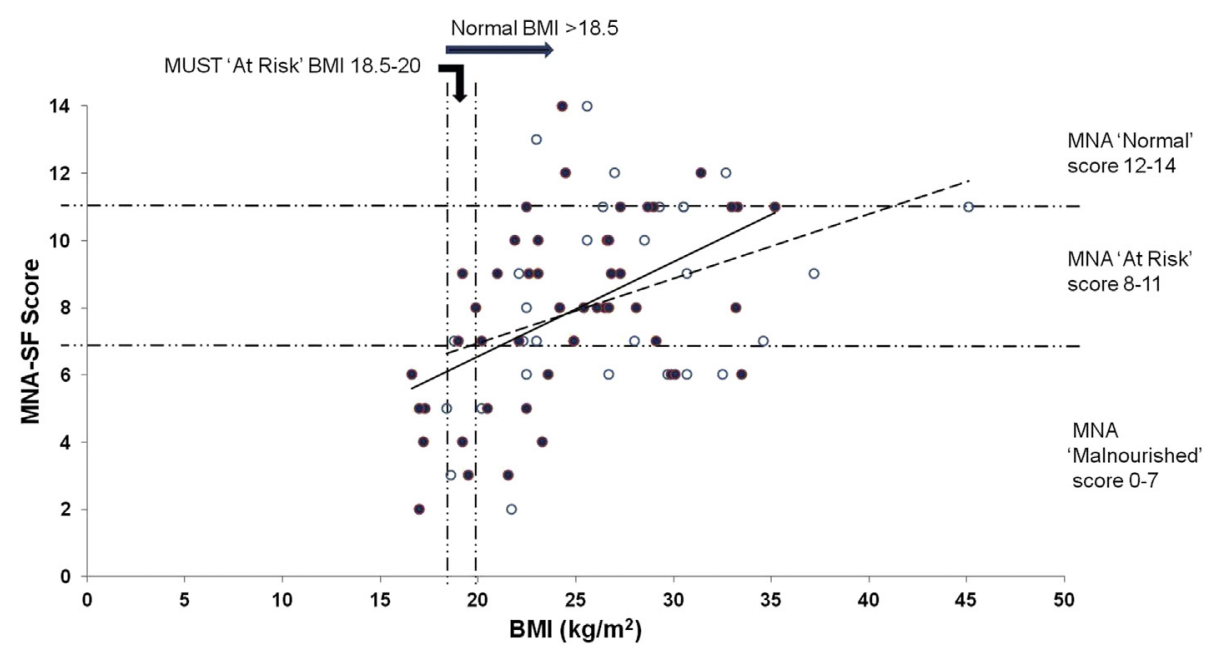

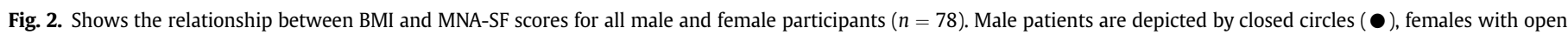
circles $(O)$, and trend lines included (males-unbroken line and females, broken line). Areas of nutritional risk by MUST and MNA are indicated.

Fig. 3A. Correlations of FFMI and MNA-SF score were; group $(r=0.284, P=0.022)$, males $(r=0.417, P=0.006)$, and females ( $r=0.98, P=0.648)$, see Fig. 3 B.

FFMI values were then compared against reference data from Schutz et al. [20]. and Coin et al. [21]. and 5th-10th percentile region used to detect low FFMI and then up to $\sim 25$ th for "borderline/ at risk' FFMI in patients; and corresponding count of matches for 'high risk'/'malnourished' and 'at risk' nutritional screening categories by MUST and MNA-SF calculated (Table 2). Analysis of FFMI categories for the male and female groups (using a $2 \times 2$ table format with normal FFMI and low/borderline FFMI), found significant differences in categorisation whereby males have higher prevalence of low/lower FFMI grouping compared to females $(P<0.05)$

\subsection{Fat mass and fat mass index}

FM and FMI for all 66 participants can be seen in Table 1B. Correlations of FMI and BMI were; group $(r=0.614, P<0.0001)$, males $(r=0.787, P<0.0001)$, and females $(r=0.91, P<0.0001)$, see Fig. 3C. Correlations of FMI and MNA-SF score were; group $(r=0.422, P=0.0004)$, males $(r=0.441, P=0.003)$, and females $(r=0.332, P=0.113)$, see Fig. 3D. Using $\sim 10$ th percentile for FMI from Schutz et al. $\left(<4.5 \mathrm{~kg} / \mathrm{m}^{2}\right) ; 12$ males $(12 / 42=29 \%)$ had low $/$
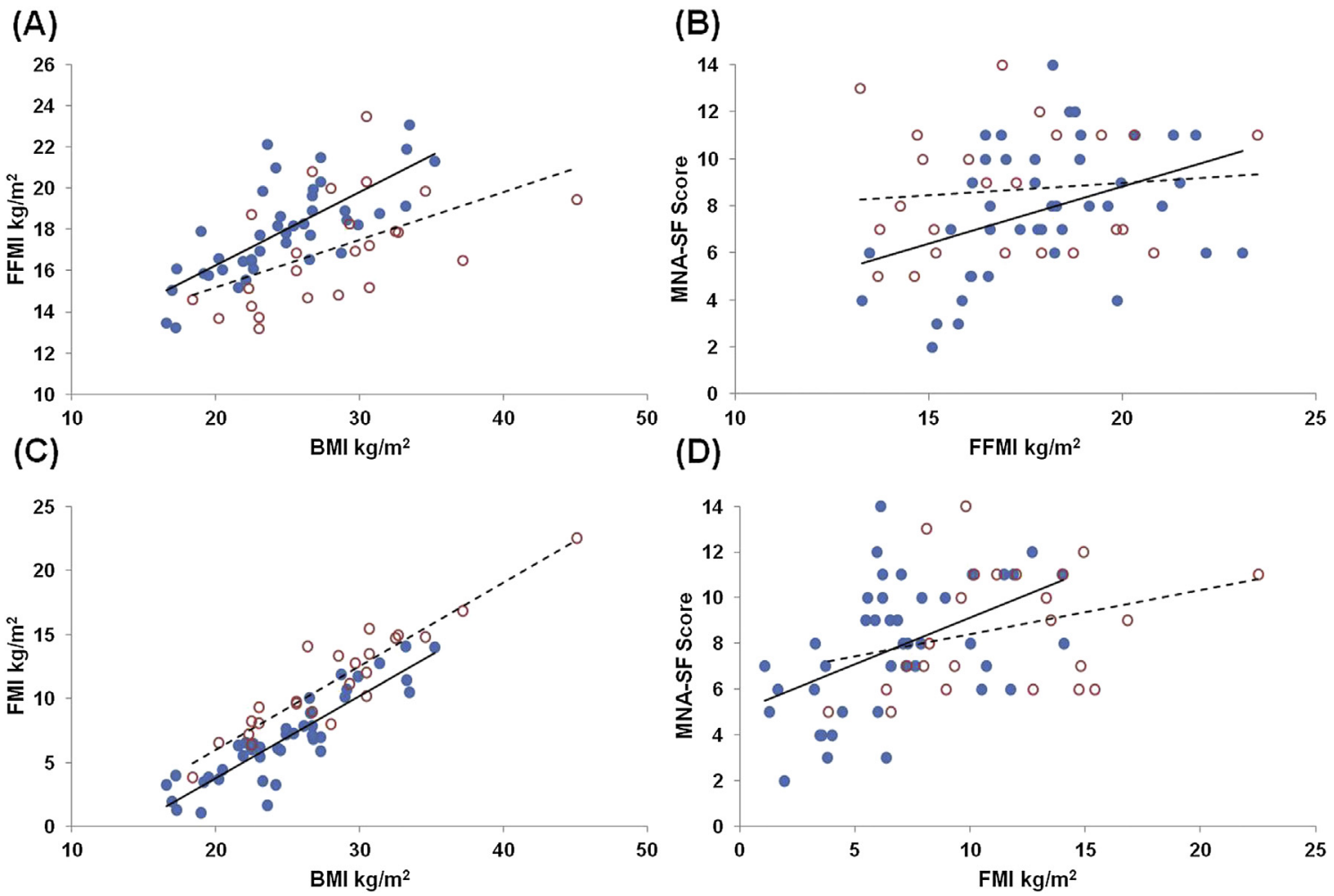

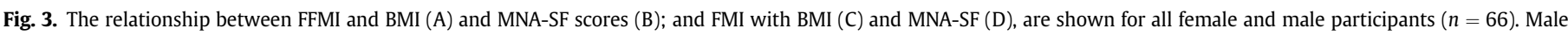
patients are depicted by closed circles $(\bullet)$, females with open circles $(O)$, and trend lines included (males-unbroken line and females, broken line). 
Table 2

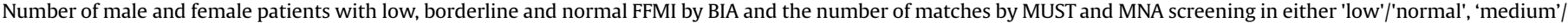
'at risk' or 'high risk'/'malnourished'category scoring.

\begin{tabular}{|c|c|c|c|c|c|c|}
\hline \multirow[b]{3}{*}{ Number of patients } & \multicolumn{3}{|l|}{ Males $(n=42)$} & \multicolumn{3}{|c|}{ Females $(n=24)$} \\
\hline & Low FFMI ${ }^{\mathrm{a}}\left(<17 \mathrm{~kg} / \mathrm{m}^{2}\right)$ & $\begin{array}{l}\text { Borderline/ } \\
\text { at risk }{ }^{\mathrm{a}}\left(17-18.3 \mathrm{~kg} / \mathrm{m}^{2}\right)\end{array}$ & Normal $\left(>18.3 \mathrm{~kg} / \mathrm{m}^{2}\right)$ & $\begin{array}{l}\text { Low FFMI } \\
\left(<13.7 \mathrm{~kg} / \mathrm{m}^{2}\right)\end{array}$ & $\begin{array}{l}\text { Borderline/at risk } \\
\left(13.7-15 \mathrm{~kg} / \mathrm{m}^{2}\right)\end{array}$ & Normal $\left(>15 \mathrm{~kg} / \mathrm{m}^{2}\right)$ \\
\hline & $17 / 42(40 \%)$ & $9 / 42(21 \%)$ & $16 / 42(38 \%)$ & $3 / 24(13 \%)$ & $4 / 24(17 \%)$ & $17 / 24$ \\
\hline MUST - 'low risk' & $9 / 17(53 \%)$ & $8 / 9(89 \%)$ & $14 / 16(87.5 \%)$ & $2 / 3(66 \%)$ & $3 / 4(75 \%)$ & $17 / 24(100 \%)$ \\
\hline MUST - 'medium risk' & $1 / 17(6 \%)$ & $1 / 9(11 \%)$ & $1 / 16(6.25 \%)$ & $1 / 3(33 \%)$ & $0 / 4(0 \%)$ & $0 / 17(0 \%)$ \\
\hline MUST - 'high risk' & $7 / 17(41 \%)$ & $0 / 9(0 \%)$ & $1 / 16(6.25 \%)$ & $0 / 3(0 \%)$ & $1 / 4(25 \%)$ & $0 / 17(0 \%)$ \\
\hline MNA - 'normal' & $0 / 17(0 \%)$ & $1 / 9(11 \%)$ & $2 / 16(12.5 \%)$ & $1 / 3(33 \%)$ & $0 / 4(0 \%)$ & $2 / 17(12 \%)$ \\
\hline MNA - 'at risk' & $6 / 17(35 \%)$ & $4 / 9(44 \%)$ & $10 / 16(62.5 \%)$ & $0 / 3(0 \%)$ & $3 / 4(75 \%)$ & $4 / 17(23.5 \%)$ \\
\hline MNA - 'malnourished' & $11 / 17(65 \%)$ & $4 / 9(44 \%)$ & $4 / 16(25 \%)$ & $2 / 3(66 \%)$ & $1 / 4(25 \%)$ & $11 / 17(64.5 \%)$ \\
\hline
\end{tabular}

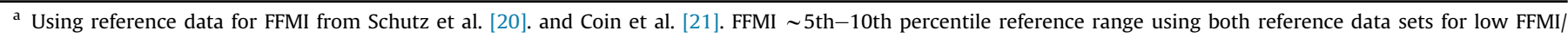
malnourished and $\sim 25$ th percentile for 'at risk'.

depleted FMI. All 12 were classified by MNA as either 'high risk'/ 'malnourished' (11/12, 92\%) or 'at risk' (1/12, 8\%). 6/12 (50\%) were classified as 'high risk' by MUST and $1 / 12$ ( $8 \%$ ) 'at risk'. No females were categorised as having low/very low FMI.

\subsection{Body cell mass and body cell mass index}

BCM and BCMI for all 66 participants can be seen in Table 1B. Correlations of BCMI and BMI were; group $(r=0.861, P<0.0001)$, males $(r=0.945, P<0.0001)$, and females $(r=0.895, P<0.0001)$. Correlations of BCMI and FFMI were; group $(r=0.579, P<0.0001)$, males $(r=0.85, P<0.0001)$, and females $(r=0.567, P<0.004)$, see Supplementary Fig. 1 in Appendices. Correlations of BCMI and MNA-SF score were; group $(r=0.46, P=0.0001)$, males $(r=0.483$, $P=0.01)$, and females $(r=0.345, P=0.99)$.

\section{Discussion}

This study investigated the use of the nutritional screening tools MUST and MNA-SF in a group of frail older hospital patients and showed clear significant differences in the group categorisation of malnutrition risk by the two tools $(P<0.000001)$. The MUST consistently scored patients within a low risk category whereas the MNA-SF scored most within 'at risk' and 'malnourished'/high risk categories (Table 1, Fig. 1). Parallel-forms reliability analysis found a poor match and reliability between the two tests. This is an issue that requires further investigation as nutritional risk categorisation has a significant impact on future clinical decisions regarding diet and nutrition in older patients on hospital wards. Recent studies showing similar MUST-MNA scoring patterns include in geriatric outpatients from the Netherlands [23], and a study in a UK care home [24].

Specific reasoning for the differences in categorisation could be due to the following factors: It is recognised that weight loss in patients increases morbidity and mortality, but accurately gauging recent weight loss in frail older people as they are admitted to a hospital ward can be practically difficult to assess- especially if the person is confused and/or has other pathological neuropsychological problems. In comparison to the MUST, the MNA has a graded scale and point category based upon weight loss e.g. it has a 'not known' category. In addition, the MNA also subjectively questions food intake over the past 3 month period, whereas the MUST requires specifically a combination of 'presence of acute disease and no nutritional intake for 5 days'.

The MNA also uses a higher grading scale for BMI compared to the MUST (which conforms to WHO guidelines, i.e. $<18.5 \mathrm{~kg} / \mathrm{m}^{2}$ is underweight and $18.5-20 \mathrm{~kg} / \mathrm{m}^{2}$, 'at risk'). The BMI of the participants in this study (Tables $1 \mathrm{~A}$ and $\mathrm{B}$ ) were predominantly within the normal and overweight ranges.
There were correlations of body weight and BMI with MNA-SF score and the spread and relationship can be viewed clearly in Fig. 2, with a high proportion of patients being categorised as malnourished or 'at risk'. This is an important finding as current research has indicated consistently that older people with higher BMI scores (including overweight and obese) have lower morbidity and mortality rates compared to those with lower BMI ranges, indicating a potential 'body mass index/obesity paradox' [25-29]. Beck and Ovesen [29], argued that the cut-off points for indicating nutritional risk in the elderly should be $24 \mathrm{~kg} / \mathrm{m}^{2}$ and a healthy BMI range should be raised from 20 to 25 to $24-29 \mathrm{~kg} / \mathrm{m}^{2}$.

The BIA readings for fat free mass index (FFMI), fat mass index (FMI) and body cell mass index (BCMI) showed significant correlations with BMI and MNA-SF scoring for all male and group correlations; however, for the female group there were similar significant correlations with BMI but not with MNA-SF.

Using reference percentiles for FFMI from Schutz et al. [20], and Coin et al. [21], patients with low and borderline FFMI were calculated and corresponding matches with MUST/MNA-SF categorisation noted (Table 2). This data supports the use of MNA-SF as the better tool to identify 'at risk' categories of malnutrition. It should also be clearly noted from Fig. 3A, the number of patients with either a low-malnourished FFMI or borderline FFMI despite having a normal or overweight BMI (by WHO and MUST), also giving strength to a BMI paradox concept.

Another particularly important measure of nutritional status is the FMI as recent evidence by Bouillanne et al. suggests that the fat mass compartment is protective of morbidity and mortality in older hospital patients [22], and in their study patients with higher BMI and FMI levels had better outcomes compared to those with lower FMI values (whereas the FFM, BCM and skeletal muscle compartments did not predict outcomes in this study). The scientific rationale would be that fat mass is essentially an energy reserve and may potentially act in a protein-sparring manner in periods of under-nutrition and stress (e.g. and potentially in older age frailty), and be partially a basis on which the BMI paradox exists in older people. FMI values in this study indicated that $29 \%$ men had low/ malnourished FMI and all were classified by MNA as either 'malnourished' (92\%) or 'at risk' (8\%); whereas 50\% of the low FMI males were classified as 'high risk' by MUST and $8 \%$ 'at risk', again underlining the use of the MNA-SF. No females were categorised as having low/very low FMI.

It can be argued that the BIA equations are not sufficiently accurate in frail older hospital patients, which we fully acknowledge. However, it is felt that the best available tools and BIA equations were used based upon the current research evidence. Findings from Lupoli et al., demonstrated good accuracy of the BIA Kyle equation for FFM using DEXA as a comparative method in underweight $\left(<20 \mathrm{~kg} / \mathrm{m}^{2}\right)$ frail male elderly patients, but interestingly not in 
females [30]. Careful inspection of our BIA data and the BMI-MNA results shows higher variability in the female group and lower prevalence of low FFMI/FMI/BCMI/malnutrition when screening by BIA- which may be accounted for by the following factors; 1) the study group was too small (24 included in BIA results); 2) In addition to being a smaller group the mean BMI was higher in females than for males and the mean levels of FFMI and BCMI were quite similar to males (Tables $1 \mathrm{~A}$ and $\mathrm{B}$ ). The general nutritional screening scores were also better for women than men. 3) There were concerns with the accuracy of the BIA in older females similar to others $[19,30]$ and it was also considered whether the reference cut-off points were appropriate for a UK population. To further analyse the pattern, BCMI data was inspected and compared to BMI and FFMI (Suppl. Fig. 1). This may potentially indicate significant ECW hydration perturbations possibly taking place, although this cannot be verified. We found it unlikely that only $13 \%$ (3/24) were malnourished from the BIA FFMI data (compared to $46 \%$ (11/24) by MNA-SF, Supplementary Table 1, Appendices). Gallagher et al., found in healthy older women the FFM compartment (using DEXA) stays relatively constant in ageing compared to significant decreases in men, although both men and women had significant decreases in skeletal muscle mass and BCM [6].

While the study group is relatively small, the findings if repeatable in larger studies would have far-reaching clinical implications. This study shows high discordance between MUST and MNA-SF scoring in frail older hospital inpatients. The BIA data supports the use of the MNA-SF as a more incisive tool in this study. This may bring into question the use of the MUST as the gold standard for assessing malnutrition risk in frail older inpatients in the UK, and may suggest that a high proportion of patients either malnourished or at risk of malnutrition may go currently unreported.

\section{Funding}

There was no funding source.

\section{Statement of authorship}

AS was the lead author and designated study Chief Investigator, DS was the designated clinical Principal Investigator; DB was a clinical co-investigator involved in data collection and critical input into both the study and manuscript preparation.

\section{Conflict of interest}

The authors declare that there are no conflicts of interest.

\section{Acknowledgements}

The authors wish to acknowledge the ward staff at Lincoln County hospital during the study.

\section{Appendix A. Supplementary data}

Supplementary data related to this article can be found at http:// dx.doi.org/10.1016/j.clnu.2014.04.013.

\section{References}

[1] Norman K, Pichard C, Lochs H, Pirlich M. Prognostic impact of disease-related malnutrition. Clin Nutr 2008;27:5-15.
[2] Sullivan DH, Patch GA, Walls RC, Lipschitz DA. Impact of nutrition status on morbidity and mortality in a select population of geriatric rehabilitation patients. Am J Clin Nutr 1990;51:749-58.

[3] Russell CA, Elia M. The skeleton in the closet: malnutrition in the community, malnutrition in the UK: where does it begin? Proc Nutr Soc 2010;69:465-9.

[4] Morley JE. Anorexia of aging: physiologic and pathologic. Am J Clin Nutr 1997;66:760-73.

[5] Kyle UG, Genton L, Hans D, Karsegard L, Slosman DO, Pichard C. Age-related differences in fat-free mass, skeletal muscle, body cell mass and fat mass between 18 and 94 years. Eur J Clin Nutr 2001;55:663-72.

[6] Gallagher D, Ruts E, Visser M, Heshka S, Baumgartner RN, Wang J, et al. Weight stability masks sarcopenia in elderly men and women. Am J Physiol Endocrinol Metab 2000;279:E366-75.

[7] Cruz-Jentoft AJ, Baeyens JP, Bauer JM, Boirie Y, Cederholm T, Landi F, et al Sarcopenia: European consensus on definition and diagnosis. Report of the European Working Group on Sarcopenia in Older People. Age Ageing 2010;39: 412-23.

[8] Fried LP, Tangen CM, Walston J, Newman AB, Hirsch C, Gottdiener J, et al Frailty in older adults: evidence for a phenotype. J Gerontol A Biol Sci Med Sci 2001:56:M146-56.

[9] Morley JE. Undernutrition in older adults. Fam Pract 2012;29:i89-93.

[10] Muscaritoli M, Anker SD, Argilé s J, Aversa Z, Bauer JM, Biolo G, et al. Consensus definition of sarcopenia, cachexia and pre-cachexia: joint document elaborated by Special Interest Groups (SIG) "cachexia-anorexia in chronic wasting diseases" and "nutrition in geriatrics". Clin Nutr; 2010. http:// dx.doi.org/10.1016/j.clnu.2009.12.004.

[11] Bauer JM, Sieber CC. Sarcopenia and frailty: a clinicians controversial point of view. Exp Gerontol 2008;2008(43):674-8.

[12] Kondrup J, Allison SP, Elia M, Vellas B, Plauth M. ESPEN Guidelines for nutritional screening 2002. Clin Nutr 2003:22(4):415-21.

[13] Elia M. Screening for malnutrition: a multidisciplinary responsibility, development and use of the malnutrition universal screening tool ('MUST') for adults. Redditch, United Kingdom: BAPEN; 2003.

[14] Vellas B, Villars H, Abellan G, Soto ME, Rolland Y, Guigoz Y, et al Overview of the $\mathrm{MNA}^{\circledR}$ - its history and challenges. J Nutr Health Aging 2006;10:456-65.

[15] Rubenstein LZ, Harker JO, Salva A, Guigoz Y, Vellas B. Screening for undernutrition in geriatric practice: developing the short-form mini nutritional assessment (MNA-SF). J Geront 2001:56A:M366-77.

[16] Kaiser MJ, Bauer JM, Ramsch C, Uter W, Guigoz Y, Cederholm T, et al. MNAInternational Group. Validation of the mini nutritional assessment short-form (MNA-SF): a practical tool for identification of nutritional status. J Nutr Health Aging 2009;13(9):782-8.

[17] Kyle UG, Bosaeus, De Lorenzo AD, Deurenberg P, Elia M, Gomez JM, et al ESPEN Guidelines. Bioelectrical impedance analysis - part I: review of principles and methods. Clin Nutr 2004;23:1226-43.

[18] Piccoli A, Nigrelli S, Caberlotto A, Bottazzo S, Rossi B, Pillon L, et al. Bivariate normal values of the bioelectrical impedance vector in adult and elderly populations. Am J Clin Nutr 1995;61:269-70.

[19] Kyle UG, Genton L, Karsegard L, Slosman DO, Pichard C. Single prediction equation for bioelectrical impedance analysis in adults aged 20-94. Nutrition 2001:17:248-53.

[20] Schutz Y, Kyle UUG, Pichard C. Fat-free mass index and fat mass index percentiles in Caucasians aged 18 - 98 y. Int J Obes Relat Metab Disord 2002;26: 953-60.

[21] Coin A, Sergi G, Minicuci N, Giannini S, Barbiero E, Manzato E, et al. Fat-free mass and fat mass reference values by dual-energy X-ray absorptiometry (DEXA) in a 20-80 year-old Italian population. Clin Nutr 2008;27:87-94.

[22] Bouillanne O, Dupont-Belmont C, Hay P, Hamon-Vilcot B, Cynober L, Aussel C, et al. Fat mass protects hospitalized elderly persons against morbidity and mortality. Am J Clin Nutr 2009;90:505-10.

[23] Heernels IM, Janse A, de Vries JHM, de Groot CPGM. Nutritional assessment of geriatric outpatients using MNA and MUST screening tools. J Aging Res Clin Pract 2013;2(1):46-50.

[24] Slee A. Estimating nutritional status in a small cohort of elderly care home residents using MUST, MNA and bioelectrical impedance phase angle and vector analysis. J Aging Res Clin Pract 2013:2(1):65-70.

[25] Mattila K, Haavisto M, Rajala S. Body mass index and mortality in the elderly Br Med J 1986;292:867-8.

[26] Flodin L, Svensson S, Cederholm T. Body mass index as a predictor of 1-year mortality in geriatric patients. Clin Nutr 2000;19(2):121-5.

[27] Weiss A, Beloosesky Y, Boaz M, Yalov A, Kornowski R, Grossman E. Body mass index is inversely related to mortality in elderly subjects. J Gen Intern Med 2007:23(1):19-24.

[28] Kaiser R, Winning K, Uter W, Volkert D, Lesser S, Stehle P, et al. Functionality and mortality in obese nursing home residents: an example of 'risk facto paradox'? J Am Med Dir Assoc 2010;11(6):428-35.

[29] Beck AM, Ovesen L. At which body mass index and degree of weight loss should hospitalised elderly patients be considered at nutritional risk? Clin Nutr 1998;17(5):195-8.

[30] Lupoli L, Sergi G, Coin A, et al. Body composition in underweight elderly subjects: reliability of bioelectrical impedance assessment. Clin Nutr 2004;23: $1371-80$. 